\title{
Surgical treatment of primary disease for penile squamous cell carcinoma: A Surveillance, Epidemiology, and End Results database analysis
}

\author{
YAO ZHU ${ }^{1,2 *}$, WEI-JIE GU ${ }^{1,2 *}$, HONG-KAI WANG ${ }^{1,2}$, CHENG-YUAN GU $^{1,2}$ and DING-WEI YE ${ }^{1,2}$ \\ ${ }^{1}$ Department of Urology, Fudan University Shanghai Cancer Center; ${ }^{2}$ Department of Oncology, \\ Shanghai Medical College, Fudan University, Shanghai 200032, P.R. China
}

Received July 30, 2014; Accepted March 27, 2015

DOI: $10.3892 / 01.2015 .3221$

\begin{abstract}
Current guidelines recommend penile sparing surgery (PSS) for selected penile cancer cases. The present study described the use of PSS in a population-based cohort, and also examined the role of PSS on penile cancer-specific mortality (PCSM). Data from the Surveillance, Epidemiology, and End Results (SEER) database were used to identify individuals that were diagnosed with penile squamous cell carcinoma between 1998 and 2009 and treated with surgery. Patients were sorted into two groups: Local tumor excision (LTE) and partial/total penectomy (PE). Factors associated with the receipt of LTE and PCSM following LTE were examined. In addition, PCSM was compared between LTE and PE following propensity score matching. Of the 1,292 eligible patients, 24.2\% underwent LTE. For stage T1 disease, the rates of LTE increased moderately from 29 to $40 \%$ over the last decade. Following multivariate analyses, young age, African descent, a tumor size of $<3 \mathrm{~cm}$ and stage $\mathrm{T} 1$ disease were identified to positively influence the receipt of LTE. With a median follow-up period of 55 months, the four-year PCSM rate was $9.8 \%$ in patients treated with LTE. Older age, a tumor size of $3-4 \mathrm{~cm}$ and regional/distant disease (SEER stage) were significant predictors of PCSM. Furthermore, in matched cohorts with stage $\mathrm{T} 1$ disease, the four-year PCSM rates were 8.9 and $10.0 \%$ for patients that received LTE or $\mathrm{PE}$, respectively $(\mathrm{P}=0.93)$. In conclusion, underuse of PSS is pronounced in the general community with significant age and ethnicity disparities. The current population-based study provides evidence supporting the oncological safety of PSS compared with PE in early-stage disease.
\end{abstract}

Correspondence to: Dr Ding-Wei Ye, Department of Urology, Fudan University Shanghai Cancer Center, 270 Dong'an Road, Shanghai 200032, P.R. China

E-mail:dwye.shca@gmail.com

*Contributed equally

Key words: penile neoplasms, SEER program, surgery, survival, multivariate analysis, prognosis

\section{Introduction}

Surgery is the mainstay of treatment for penile squamous cell carcinoma (SCC). Partial or total penectomy (PE) has historically been considered the standard treatment for invasive disease (1). Although the local control rate of PE is 95\% (1), it has a significant negative impact on the patient's sexual function, quality of life, social interactions, self-image and self-esteem (2). During the past decade, there has been a change in the management of primary tumors with an emphasis on penile sparing surgery (PSS) (3). This change has been driven by an improved understanding of the biology of the disease (4), quality improvements in pathological evaluation and continuous refinements of surgical techniques (5). PSS has been previously reported to produce excellent cosmetic and functional results without sacrificing oncological outcomes in certain patients with early-stage penile tumors (3,6-9). Accordingly, an organ-sparing approach has been recommended for patients with stage T1 disease, according to the 2009 TNM clinical and pathological classification system (10), in national and international guidelines (11-13). Stage T1 penile tumors are classified as tumors which have invaded the subepithelial connective tissue, without invasion of the corpus spongiosum or corpora cavernosa (10).

Despite the evolution of conservative surgery at academic centers, the national practice pattern of surgical treatment for penile SCC in the United States (US) is largely unknown. Therefore, the aims of the current study were to examine data from the most recent Surveillance, Epidemiology and End Results (SEER) cancer registry (14), and to elucidate whether there are disparities in the use of PSS. Since only a limited number of reports exist regarding the oncological safety of PSS, the current study aimed to examine the penile cancer-specific survival following conservative surgery in a population-based setting, and to compare oncological outcomes between PSS and PE in stage $\mathrm{T} 1$ disease.

\section{Materials and methods}

Participants and variables. The SEER program was used to identify patients who received surgical treatment for primary invasive penile SCC between 1998 and 2009. The 
population-based database included 18 cancer registries and covered $\sim 28 \%$ of the US population. Since the study used a public set of identified data, it was exempted from institutional review boards.

Case listing. Case listing was generated using codes specific for primary site and morphology, which included the following: The International Classification of Disease for Oncology 2nd edition (ICD-O-2; codes, C60.0-60.9) and 3rd edition (ICD-O-3; codes, 8070-8076) for histological subtype (SCC type). The sample was limited to patients with adequate information and a preliminary cohort of 1,293 patients diagnosed with invasive penile SCC was identified. A patient who underwent local tumor destruction was excluded. Therefore, this process yielded a sample comprising 1,292 eligible patients.

Surgical procedures. The surgical procedures for primary disease were identified and separated into two groups: Local tumor excision (LTE) and PE. The SEER database was used to retrieve demographic and disease characteristics, including age, ethnicity, marital status, year of diagnosis, tumor stage (T-stage), primary tumor size, SEER stage and grade. T-stages were assigned according to the American Joint Committee on Cancer 6th staging system (15). SEER stage is a simplified version of stage defining the extent of the disease (localized, regional and distant). Survival time was measured as the interval from the date of diagnosis until the date of mortality or until the last follow-up. Mortalities from penile cancer were coded as penile cancer-specific mortality (PCSM) and all other mortalities were considered as other-cause mortality (OCM).

Statistical analysis. Continuous data are presented as the median [interquartile range (IQR)] and categorical data are presented as proportions. The $\chi^{2}$ test for trend was used to evaluate whether there was a linear trend in the proportions. Multivariate logistic regression analysis was used to evaluate the adjusted associations between covariables and utilization of LTE.

A substantial proportion of patients with penile SCC succumb to the disease as a result of competing causes of mortality, such as ischemic heart disease, stroke and diabetes (16). Competing risk analysis was used to account for the effect of OCM and provide unbiased estimates of PCSM. The cumulative incidence was plotted to graphically depict PCSM and OCM. Gray's test was used to assess the statistical significance of a prognostic factor in a cumulative incidence analysis (17). Multivariable competing risk regression analysis was used to evaluate the adjusted effects of covariates on PCSM, as proposed by Fine and Gray (18).

In order to enable balanced comparisons between LTE and PE, propensity score matching was used to adjust for the inherent selection bias within observational data (19). Using propensity score matching, randomized trials can be statistically reproduced by balancing the characteristics of different treatment groups. The propensity to undergo LTE was calculated using a multivariable logistic regression model that adjusted significant confounders. In addition, the nearest neighbor method matching, with a caliper width of 0.2 of the standard deviation of the logit, was used to match cases. The
Table I. Demographic and disease characteristics of 1,292 patients with penile squamous cell carcinoma (1998-2009).

\begin{tabular}{lc}
\hline Characteristics & $\mathrm{n}(\%)$ \\
\hline Age, years (median, interquartile range) & $67(57-77)$ \\
Ethnicity & \\
Caucasian & $1,107(85.7)$ \\
African descent & $119(9.2)$ \\
Other & $66(5.1)$ \\
Marital status & \\
Married & $813(62.9)$ \\
Single & $479(37.1)$ \\
Year of diagnosis & \\
1988-2000 & $250(15.4)$ \\
2001-2003 & $449(27.7)$ \\
2004-2006 & $434(26.7)$ \\
2007-2009 & $490(30.2)$ \\
Tumor stage & \\
T1 & $699(54.1)$ \\
T2 & $381(29.5)$ \\
T3-4 & $212(16.4)$
\end{tabular}

Primary tumor size, $\mathrm{cm}$

$<1$

$106(8.2)$

$1-1.9$

$259(20.0)$

$2-2.9$

$304(23.5)$

3-3.9

$261(20.2)$

$\geq 4$

$362(28.0)$

SEER stage

Localized

$706(54.6)$

Regional

$550(42.6)$

Distant

Tumor grade

Grade I

$366(28.3)$

Grade II

$639(49.5)$

Grade III-IV

$287(22.2)$

Treatment of primary disease

Local tumor excision

313 (24.2)

Partial penectomy

801 (62.0)

Total penectomy

178 (13.8)

SEER, Surveillance, Epidemiology and End Results program.

standardized difference measure was also used to assess how closely the PE patients matched the LTE cases.

All the analyses were performed using $\mathrm{R}$ software (version 3.0.0; http://www.r-project.org.). P-values were two-tailed and $\mathrm{P}<0.05$ was considered to indicate a statistically significant difference.

\section{Results}

Clinical characteristics of patients. The descriptive characteristics of the 1,292 eligible patients with penile SCC are presented 
A

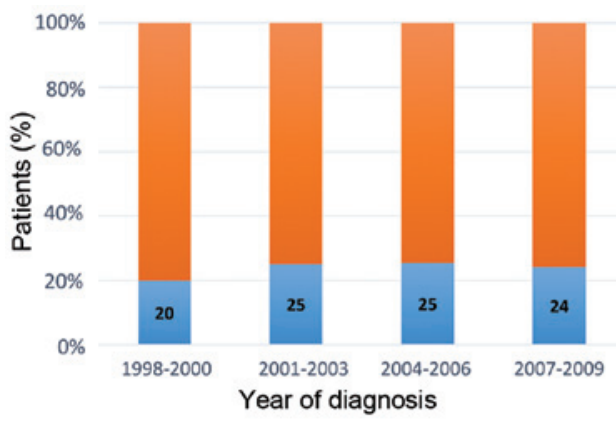

C $100 \%$

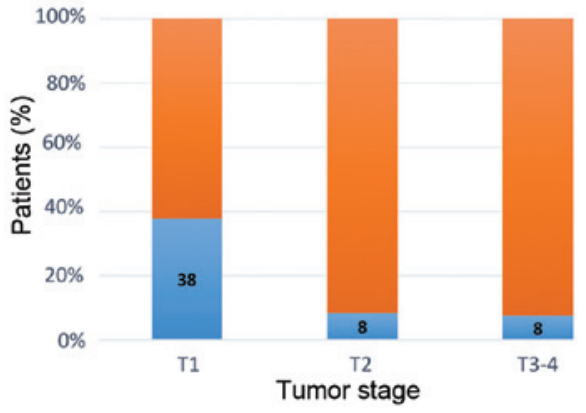

B

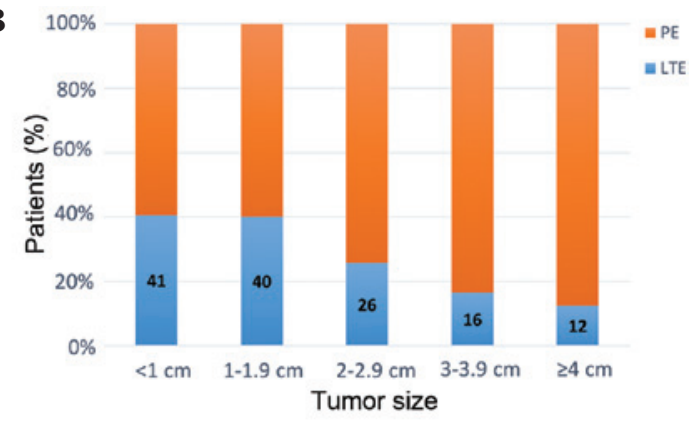

D

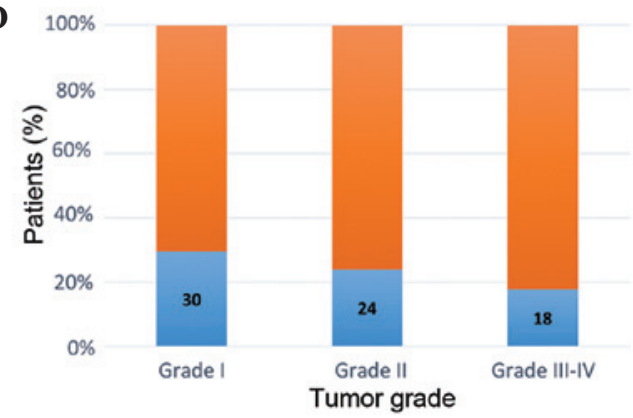

Figure 1. Distribution of LTE vs. PE in the eligible patients ( $\mathrm{n}=1,292)$, stratified according to (A) year of diagnosis, (B) primary tumor size, (C) tumor stage and (D) tumor grade. LTE, local tumor excision; PE, partial/total penectomy.

in Table I. The median age was 67 years, while the majority of patients were Caucasian (85.7\%), married (62.9\%) and diagnosed with $\mathrm{T} 1$ disease (54.1\%). Of these patients, 313 (24.2\%) underwent LTE and 979 (75.8\%) received partial or total PE.

Distribution of LTE and PE. Fig. 1 illustrates the distribution of LTE and PE stratified according to the year of diagnosis, primary tumor size, T-stage and tumor grade. Increased LTE utilization rates were observed in smaller tumors, lower T-stage and lower tumor grade (all $\mathrm{P}<0.05$ ). The overall use of LTE in the general population was similar throughout the study period $(\mathrm{P}=0.53)$.

Rate of LTE within stage T1 disease patients. The rate of LTE within patients with stage $\mathrm{T} 1$ disease was further investigated (Fig. 2). Among the 699 stage T1 patients, 265 (37.9\%) were treated with LTE. The rate of LTE increased moderately from 29 to $40 \%$ over the study period, however this increase was not statistically significant ( $\mathrm{P}=0.10$; Fig. $2 \mathrm{~A})$. In addition, patients with smaller tumors were more likely to receive LTE $(\mathrm{P}<0.01$; Fig. 2B). However, lower tumor grade was not associated with a higher rate of LTE ( $\mathrm{P}=0.56$; Fig. $2 \mathrm{C})$.

Using multivariate analyses, the adjusted associations between individual characteristics and the use of LTE was assessed. Table II demonstrates that patients treated with LTE were younger, more often of African descent, with tumor size of $<3 \mathrm{~cm}$ and with stage $\mathrm{T} 1$ disease (all $\mathrm{P}<0.01$ ). Unmarried men were more likely to select PE treatment than LTE, whereas married men were more likely to receive LTE than $\mathrm{PE}$, however, no signficant difference was identified $(\mathrm{P}=0.08)$. By contrast, SEER stage and tumor grade were not independent predictors of conservative surgery.

Survival outcomes. The survival outcomes in 313 patients who received LTE were further evaluated. During a

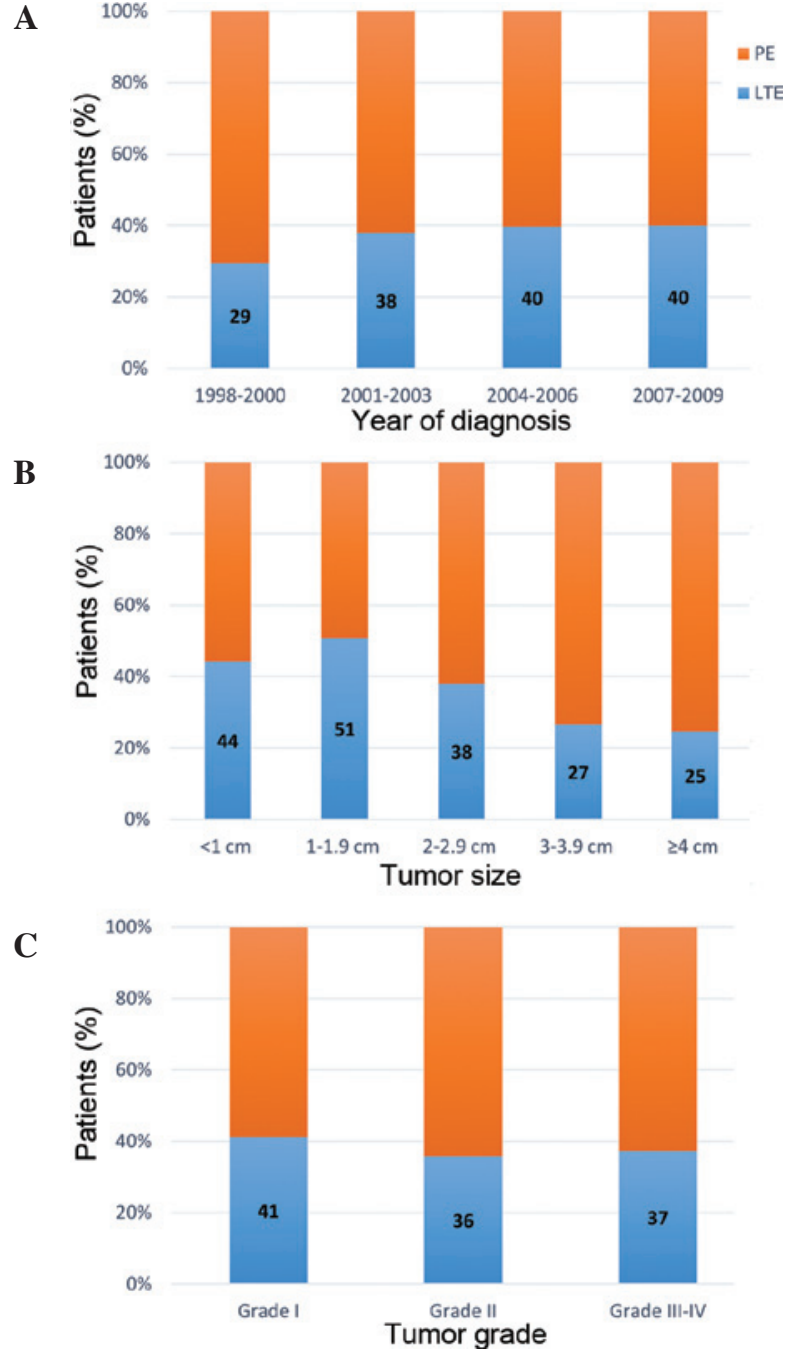

Figure 2. Distribution of LTE vs. PE in patients with stage T1 disease ( $n=699)$, stratified according to (A) year of diagnosis, (B) primary tumor size and (C) tumor grade. LTE, local tumor excision; PE, partial/total penectomy. 
Table II. Multivariate analyses of predictors for the receipt of local tumor excision in patients with penile SCC $(n=1292)$.

\begin{tabular}{|c|c|c|}
\hline Variables & Odds ratio $(95 \% \mathrm{CI})$ & P-value \\
\hline Age & $0.99(0.98-1.00)$ & $<0.01$ \\
\hline \multicolumn{3}{|l|}{ Ethnicity ${ }^{\mathrm{a}}$} \\
\hline African descent & $1.72(1.07-2.75)$ & 0.02 \\
\hline Other & $0.9(0.46-1.75)$ & 0.75 \\
\hline \multicolumn{3}{|l|}{ Marital status $^{\mathrm{b}}$} \\
\hline Unmarried & $1.30(0.97-1.75)$ & 0.08 \\
\hline \multicolumn{3}{|c|}{ Primary tumor size $^{\mathrm{c}}, \mathrm{cm}$} \\
\hline $1-1.9$ & $1.20(0.74-1.96)$ & 0.46 \\
\hline $2-2.9$ & $0.70(0.43-1.14)$ & 0.15 \\
\hline $3-3.9$ & $0.44(0.25-0.75)$ & $<0.01$ \\
\hline$\geq 4$ & $0.37(0.21-0.63)$ & $<0.01$ \\
\hline \multicolumn{3}{|l|}{ Tumor stage $^{\mathrm{d}}$} \\
\hline $\mathrm{T} 2$ & $0.18(0.10-0.31)$ & $<0.01$ \\
\hline $\mathrm{T} 3-\mathrm{T} 4$ & $0.16(0.08-0.31)$ & $<0.01$ \\
\hline \multicolumn{3}{|l|}{ SEER $_{\text {stage }}{ }^{\mathrm{e}}$} \\
\hline Regional & $1.09(0.68-1.74)$ & 0.71 \\
\hline Distant & $1.16(0.40-3.37)$ & 0.78 \\
\hline \multicolumn{3}{|l|}{ Tumor grade ${ }^{\mathrm{f}}$} \\
\hline Grade II & $0.92(0.67-1.26)$ & 0.60 \\
\hline Grade III-IV & $0.77(0.50-1.17)$ & 0.21 \\
\hline \multicolumn{3}{|l|}{ Year of diagnosis ${ }^{\mathrm{g}}$} \\
\hline 2001-2003 & $1.31(0.81-2.14)$ & 0.28 \\
\hline 2004-2006 & $1.46(0.90-2.37)$ & 0.12 \\
\hline 2007-2009 & $1.38(0.86-2.21)$ & 0.18 \\
\hline
\end{tabular}

Compared with: ${ }^{a}$ Caucasian ethnicity; ${ }^{b}$ married status; ${ }^{c}$ tumor size of <1; ${ }^{\mathrm{d}} \mathrm{T} 1$ stage; ${ }^{\mathrm{e}}$ localized tumor; ${ }^{\mathrm{f}}$ Grade I tumor; ${ }^{\mathrm{g}}$ diagnosis in 1988-2000. SCC, squamous cell carcinoma; CI, confidence interval; SEER, Surveillance, Epidemiology and End Results program.

median follow-up of 55 months (IQR, 45-65 months), 29 patients $(9.3 \%)$ succumbed to penile cancer and 79 patients $(25.2 \%)$ succumbed to another cause. Estimates of PCSM and OCM are presented in Fig. 3. Mortality in patients treated with LTE was not usually a result of the penile cancer. The risk factors associated with PCSM were investigated in this cohort (Table III). Patients who were older, with a tumor size of 3-4 cm and with regional or distant disease (SEER stage) had a significantly increased likelihood of mortality associated with penile cancer (all $\mathrm{P}<0.05$ ). Multivariate analyses were also performed to identify risk factors in males that underwent PE ( $n=979)$. By contrast, only tumor grade and SEER stage were independent predictors of PCSM (data not shown).

Risk of PCSM in T1 disease patients. Whether LTE was associated with a higher risk of PCSM was investigated in the $\mathrm{T} 1$ disease subgroup. Of these patients, 265 (37.9\%) and $434(62.1 \%)$ were treated with LTE or PE, respectively. During a median follow-up period of 59 months (IQR, 54-66 months), penile cancer was identified as the cause
Table III. Multivariate analyses of predictors of penile cancer-specific mortality in patients treated with local tumor excision $(n=313)$.

\begin{tabular}{|c|c|c|}
\hline Variables & Hazard ratio $(95 \% \mathrm{CI})$ & P-value \\
\hline Age & $1.03(1.00-1.05)$ & 0.03 \\
\hline \multicolumn{3}{|l|}{ Ethnicity $^{\mathrm{a}}$} \\
\hline African descent/Other & $0.15(0.02-1.21)$ & 0.08 \\
\hline \multicolumn{3}{|l|}{ Marital status ${ }^{\mathrm{b}}$} \\
\hline Single & $0.75(0.31-1.80)$ & 0.52 \\
\hline \multicolumn{3}{|l|}{ Primary tumor $\operatorname{size}^{c}, \mathrm{~cm}$} \\
\hline $1-1.9$ & $1.00(0.19-5.17)$ & 1.00 \\
\hline $2-2.9$ & $2.41(0.50-11.52)$ & 0.27 \\
\hline $3-3.9$ & $6.79(1.32-35.07)$ & 0.02 \\
\hline$\geq 4$ & $1.99(0.35-11.32)$ & 0.44 \\
\hline \multicolumn{3}{|l|}{ Tumor stage $^{\mathrm{d}}$} \\
\hline $\mathrm{T} 2-\mathrm{T} 4$ & $0.49(0.15-1.55)$ & 0.22 \\
\hline \multicolumn{3}{|l|}{ SEER stage ${ }^{\mathrm{e}}$} \\
\hline Regional/Distant & $4.83(1.74-13.38)$ & $<0.01$ \\
\hline \multicolumn{3}{|l|}{ Tumor grade ${ }^{f}$} \\
\hline Grade II & $0.76(0.29-2.00)$ & 0.58 \\
\hline Grade III-IV & $0.94(0.27-3.23)$ & 0.92 \\
\hline
\end{tabular}

Compared with: ${ }^{a}$ Caucasian ethnicity; ${ }^{b}$ married status; ${ }^{c}$ tumor size of $<1$; ${ }^{\mathrm{d}} \mathrm{T} 1$ stage; ${ }^{\mathrm{e}}$ localized tumor; ${ }^{\mathrm{f}}$ Grade I tumor. CI, confidence interval; SEER, Surveillance, Epidemiology and End Results program.

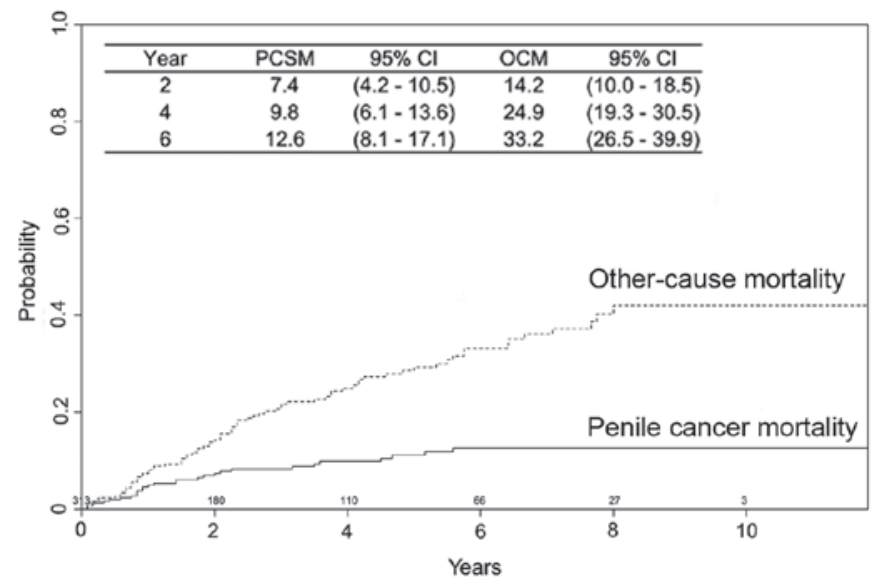

Figure 3. Cumulative incidence plot depicting PCSM and OCM rates in patients treated with local tumor excision $(n=313)$. PCSM, penile cancer-specific mortality; CI, confidence interval; OCM, other cause mortality.

of mortality in 22 patients $(8.3 \%)$ treated with LTE and 41 patients $(9.4 \%)$ treated with PE. As shown in Fig. 4, no statistically significant difference in PCSM was observed between the treatment groups $(\mathrm{P}=0.66)$. In addition, OCM was comparable for patients treated with LTE or PE $(\mathrm{P}=0.21)$. In order to reduce selection bias in the assignment of treatments, matched groups were generated using the propensity score matching method. Table IV demonstrates that significant variations of covariates were diminished following statistical 
Table IV. Propensity score matching of surgical procedures in patients with stage T1 disease.

\begin{tabular}{|c|c|c|c|c|c|}
\hline \multirow[b]{2}{*}{ Variables } & \multirow[b]{2}{*}{ Tumor excision } & \multicolumn{2}{|c|}{ Prior to matching } & \multicolumn{2}{|c|}{ Subsequent to matching } \\
\hline & & Penectomy & P-value & Penectomy & P-value \\
\hline Total number, $\mathrm{n}$ & 265 & 434 & & 265 & \\
\hline Mean age, years & 64.7 & 67.7 & $<0.01$ & 65.0 & 0.84 \\
\hline Ethnicity & & & 0.48 & & 0.21 \\
\hline Caucasian & 222 & 375 & & 229 & \\
\hline African descent & 30 & 37 & & 19 & \\
\hline Other & 13 & 22 & & 17 & \\
\hline Marital status & & & 0.53 & & 0.23 \\
\hline Married & 167 & 285 & & 181 & \\
\hline Single & 98 & 149 & & 84 & \\
\hline Tumor grade & & & 0.42 & & 0.33 \\
\hline Grade I & 100 & 143 & & 85 & \\
\hline Grade II & 122 & 219 & & 138 & \\
\hline Grade III-IV & 43 & 72 & & 42 & \\
\hline Primary tumor size, $\mathrm{cm}$ & & & $<0.01$ & & 0.92 \\
\hline$<1$ & 38 & 48 & & 44 & \\
\hline $1-1.9$ & 97 & 94 & & 88 & \\
\hline $2-2.9$ & 67 & 109 & & 68 & \\
\hline 3-3.9 & 33 & 91 & & 33 & \\
\hline$\geq 4$ & 30 & 92 & & 32 & \\
\hline SEER stage & & & 0.29 & & 0.66 \\
\hline Localized & 242 & 384 & & 238 & \\
\hline Regional/Distant & 23 & 50 & & 27 & \\
\hline Mean propensity score & 0.59 & 0.64 & $<0.01$ & 0.59 & 0.55 \\
\hline
\end{tabular}

SEER, Surveillance, Epidemiology and End Results program.

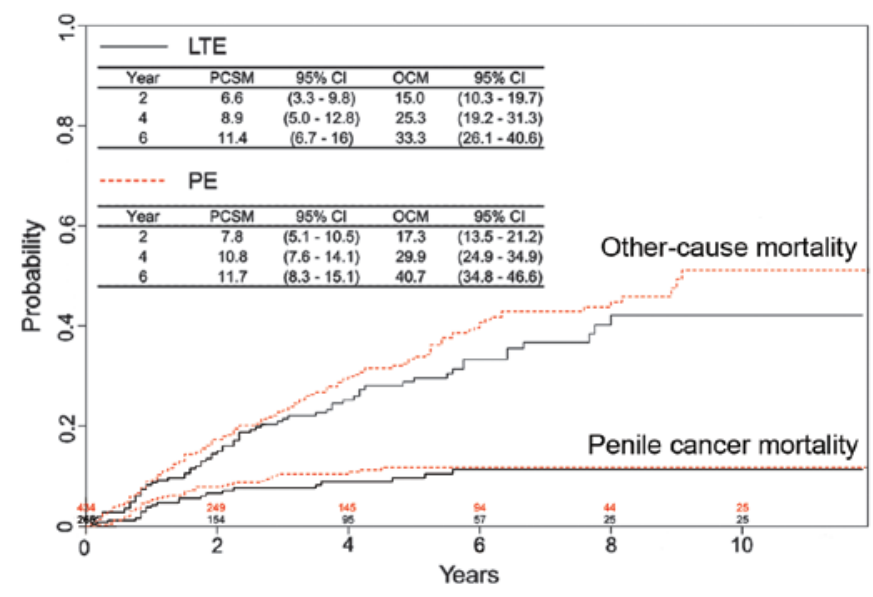

Figure 4. Cumulative incidence plot depicting PCSM and OCM rates stratified according to the surgical procedures in stage T1 patients $(n=699)$. PCSM, penile cancer-specific mortality; OCM, other-cause mortality; CI, confidence interval; LTE, local tumor excision; PE, partial/total penectomy.

processing. In the matched series, the rate of PCSM did not differ significantly between patients treated with LTE or PE, with four-year PCSM rates of 8.9 and $10.0 \%$, respectively $(\mathrm{P}=0.93$; Fig. 5).

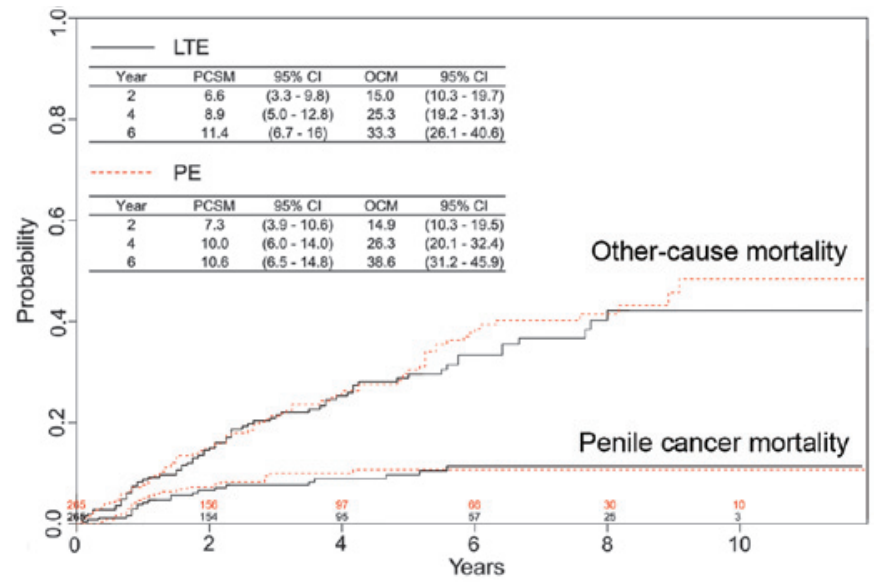

Figure 5. Cumulative incidence plot depicting PCSM and OCM rates stratified according to the surgical procedures in matched stage T1 patients $(n=530)$. PCSM, penile cancer-specific mortality; OCM, other-cause mortality; CI, confidence interval; LTE, local tumor excision; PE, partial/total penectomy.

\section{Discussion}

Over the past decade, growing evidence has indicated the safety, efficacy and benefit of PSS over PE for certain patients 
Table V. Literature review of oncological outcomes following penile-sparing surgery.

\begin{tabular}{lcccc}
\hline First author, year (ref) & Number of patients, $\mathrm{n}$ & Tumor stage $\geq$ T1, $\%$ & Follow-up, months & Local recurrence rate, $\%$ \\
\hline Smith, 2007 (3) & 72 & 100.0 & 27 (mean) & 4.2 \\
Leijte, 2008 (1) & 415 & 69.4 & 60.6 (median) & 27.7 \\
Morelli, 2009 (20) & 17 & 88.2 & 36 (mean) & 0.0 \\
Feldman, 2011 (21) & 56 & 50.0 & 60 (median) & 21.4 \\
Li, 2011 (8) & 32 & 78.1 & 26.5 (median) & 9.3 \\
O'Kane, 2011 (22) & 25 & 76.0 & 28 (mean) & 4.0 \\
Li, 2012 (23) & 12 & 25.0 & 62.5 (mean) & 8.3 \\
Philippou, 2012 (6) & 179 & 100.0 & 39 (median) & 8.9 \\
Veeratterapillay, 2012 (7) & 65 & 76.9 & 40 (median) & 6.2 \\
Total & 873 & 77.8 & & 17.8 \\
\hline
\end{tabular}

${ }^{a}$ For patients without recurrence.

with early-stage penile tumors. A total of 10 studies reported in the literature were identified, which investigated the role of conservative surgery in invasive penile cancer (Table $\mathrm{V} ; 1$, $3,6-8,20-23$ ). Local disease control was achieved in $82.2 \%$ of the cases following PSS. Although PSS was associated with an increased risk of local failure, it did not appear to compromise long-term cancer-specific survival. The five-year cancer-specific survival rate following local recurrence was $92 \%$ in two large studies $(1,6)$. In addition, sexual function, micturition and cosmetic results were generally well maintained following conservative surgery (3, 6-8,20-23). Accordingly, the use of PSS has risen dramatically at tertiary care centers and has been recommended by certain guidelines, including the European Association of Urology (EAU) Guidelines Group on Penile Cancer, as a standard surgical approach (11-13).

The surgical treatment paradigm for primary disease emphasizes the underuse of PSS in the USA. In the present study, $\geq 50 \%$ of patients with a T1 tumor of $<2 \mathrm{~cm}$ were identified to have undergone traditional radical surgery. Although the results identified that the utilization rate of LTE has increased from 29 to $40 \%$ in stage T1 disease over the last decade, PE remains the most commonly performed type of surgery for early-stage penile tumors. The reasons for this are multifactorial and may include the lack of substantial evidence for oncological safety and the technical challenges associated with PSS. The long-term outcomes of PSS have not previously been available to prove that conservative surgery provided comparable cancer-specific survival as conventional procedures $(1,6)$. Furthermore, there may be a requirement for additional training of surgical skills to safely and effectively perform PSS. Since there is no centralized management of this rare disease in North America, treatment standardization and gain of experience are slow in the community setting.

The disparities in the use of LTE in the general practice pattern were also investigated in the present study. As expected, tumor size and T-stage were strongly associated with the use of LTE. Furthermore, LTE was more frequently performed in younger patients or those of African descent.
The reason for the disparities in the use of LTE in different ages and ethnicities remains unclear. However, the same phenomenon was observed in patients choosing to receive a penile implant following treatment of prostate cancer (24). In multivariate analysis, predictors of penile implant surgery were younger and of African-American descent. Therefore, the authors speculated that physicians may have a better appreciation of the impact of PSS in young male patients and those of African-American descent (24). Further investigation of these biases is warranted, and increased education of physicians and patients may alleviate the discrepancy of care.

In the population-based cohort, the six-year PCSM rate following LTE was $12.6 \%$, which is consistent with other large studies $(1,6)$. On the contrary, mortality in long-term survivors were more likely from a cause other than penile cancer. Accounting for the influence of competing risk, the factors that predict mortality from penile cancer following conservative surgery were examined in the present study. Notably, old age and large tumor size were independent predictors of PCSM in patients treated with LTE. Therefore, these two factors may indicate an increased risk of failure to the specific treatment. Mohs et al (25) previously reported the five-year follow-up of penile cancer patients treated with micrographic surgery. Initial tumor size appeared to be an important factor in local control with $0 \%$ recurrence in males with lesions $<2 \mathrm{~cm}$ and $50 \%$ recurrence in those with lesions $>3 \mathrm{~cm}$. As the average length of the glans is $\sim 4 \mathrm{~cm} \mathrm{(26),}$ local excision of tumors with a size $>3 \mathrm{~cm}$ may result in a tumor-free margin of $<1 \mathrm{~cm}$. Agrawal et al (4) observed an absence of grade 1-2 penile SCC that extended $1 \mathrm{~cm}$ beyond the gross tumor margin; however, $25 \%$ of grade 3 lesions were histologically positive at $1 \mathrm{~cm}$ (4). In these patients with larger tumors, glansectomy with reconstruction is a better alternative to achieve satisfactory oncological and functional outcomes (27). The reason why age was a negative predictor in LTE patients is not clear. One explanation is that elderly patients may be subject to suboptimal follow-up, which is critically important following PSS. In addition, other causes of mortality may be misattributed as mortality due to penile cancer in older patients. Furthermore, the natural disease 
course of penile SCC may be more aggressive in the elderly. Epidemiology studies of vulval cancer have demonstrated a bimodal age distribution; human papillomavirus (HPV)-associated cancer manifests at an earlier age compared with HPV-unrelated cancer (28). Bezerra et al (29) reported a significantly lower HPV infection rate in elderly patients. It is possible that penile cancer in elderly patients may arise from a carcinogenesis pathway with a high degree of genetic alterations not driven by HPV (30). Since detailed follow-up data regarding tumor recurrences were not available and the SEER database does not contain further information on tumor characteristics beyond stage and grade, the explanations proposed in the present study remain speculative.

Although stage T1 disease is recommended as an appropriate indication for PSS (11-13), no direct comparison exists between LTE and PE in these patients. In the SEER database, 37.9 and $62.1 \%$ of stage T1 disease patients underwent conservative surgery or PE, respectively. Although no randomized assignment of treatment existed in the observational study, statistical processing may aid in the adjustment for differences of baseline characteristics and reduce the inherited selection bias. Therefore, the propensity score matching method was employed in the current study to mimic random allocation of treatment in stage $\mathrm{T} 1$ penile SCC. The comparisons of PCSM between matched cohorts concluded that penile cancer-specific survival following PSS was not reduced. Corroborating the findings from tertiary care centers, the results of the present study provide considerable evidence to support the generalization of conservative surgery on a large scale. In stage T1 disease, the utilization rate of LTE has improved from 29 to $40 \%$ over the last decade. Although encouraging, the changing trend remains slow compared with the similar situation in breast-sparing surgery (31). The accumulating evidence may accelerate the dissemination of PSS in early-stage penile tumors.

However, the current study is not devoid of limitations. Although the SEER program provides the largest and most comprehensive cancer registry in the USA, there are other variables not routinely collected, including anatomical features, comorbid conditions and patient preferences. These factors may account for some of the observed disparities in the use of PSS. Since the SEER program only records the first course of treatment received, adjuvant therapy for primary disease and its effect on PCSM may not be evaluated from this data. The conclusions of the present study may be biased due to excluding a proportion of patients from analyses as a result of missing information. Furthermore, there are inherent difficulties in accurately determining the types of LTE based on ambiguity in coding. Despite these limitations, the current study represents a comprehensive and contemporary analysis of the surgical treatments for primary lesions of penile cancer in the USA. The results may add value to existing knowledge since the majority of previous case studies were single-center based and thus more likely to have a selection bias. Furthermore, accumulating large cohorts from contemporary practice is challenging for rare cancer types.

In conclusion, the underuse of PSS is pronounced in the general community with significant disparities in age and ethnicity. The present population-based study provides evidence supporting the oncological safety of PSS as compared with PE in early-stage disease. Awareness of these issues may improve the quality of health care in penile cancer patients.

\section{Acknowledgements}

The authors would like to thank all the study participants, urologists and study coordinators for participating in the study.

\section{References}

1. Leijte JA, Kirrander P, Antonini N, Windahl T and Horenblas S: Recurrence patterns of squamous cell carcinoma of the penis: Recommendations for follow-up based on a two-centre analysis of 700 patients. Eur Urol 54: 161-168, 2008.

2. Maddineni SB, Lau MM and Sangar VK: Identifying the needs of penile cancer sufferers: A systematic review of the quality of life, psychosexual and psychosocial literature in penile cancer. BMC Urol 9: 8, 2009.

3. Smith Y, Hadway P, Biedrzycki O, Perry MJ, Corbishley C and Watkin NA: Reconstructive surgery for invasive squamous carcinoma of the glans penis. Eur Urol 52: 1179-1185, 2007.

4. Agrawal A, Pai D, Ananthakrishnan N, Smile SR and Ratnakar C: The histological extent of the local spread of carcinoma of the penis and its therapeutic implications. BJU Int 85: 299-301, 2000.

5. Hegarty PK, Shabbir M, Hughes B, et al: Penile preserving surgery and surgical strategies to maximize penile form and function in penile cancer: Recommendations from the United Kingdom experience. World J Urol 27: 179-187, 2009.

6. Philippou P, Shabbir M, Malone P, et al: Conservative surgery for squamous cell carcinoma of the penis: Resection margins and long-term oncological control. J Urol 188: 803-808, 2012.

7. Veeratterapillay R, Sahadevan K, Aluru P, Asterling S, Rao GS and Greene D: Organ-preserving surgery for penile cancer: description of techniques and surgical outcomes. BJU Int 110: 1792-1795, 2012.

8. Li J, Zhu Y, Zhang SL, et al: Organ-sparing surgery for penile cancer: complications and outcomes. Urology 78: 1121-1124, 2011.

9. Lont AP, Gallee MP, Meinhardt W, van Tinteren $\mathrm{H}$ and Horenblas S: Penis conserving treatment for T1 and T2 penile carcinoma: Clinical implications of a local recurrence. J Urol 176: 575-580, 2006.

10. Sobin LH, Gospodarowicz MK and Wittekind C (eds): TNM Classification of Malignant Tumours. 7 th edition. Wiley-Blackwell, Oxford, 2009.

11. Solsona E, Bahl A, Brandes SB, et al: New developments in the treatment of localized penile cancer. Urology 76 (Suppl 1): S36-S42, 2010

12. Pizzocaro G, Algaba F, Horenblas S, et al; European Association of Urology (EAU) Guidelines Group on Penile Cancer: EAU penile cancer guidelines 2009. Eur Urol 57: 1002-1012, 2010.

13. National Comprehensive Cancer Network: Clinical practice guidelines in oncology. http://www.nccn.org/professionals/ physician_gls/f_guidelines.asp. Accessed December 2, 2013.

14. National Cancer Institute: SEER*Stat Databases: November 2011 Submission. http://seer.cancer.gov/data/seerstat/nov2011/. Accessed February 4, 2014.

15. Greene FL, Page DL, Fleming ID, Fritz A, Haller DG and Morrow M: AJCC cancer staging manual. 6th edition. Springer-Verlag, Berlin, Germany, 2002.

16. Thuret R, Sun M, Abdollah F, et al: Competing-risks analysis in patients with $\mathrm{T} 1$ squamous cell carcinoma of the penis. BJU Int 111: E174-E179, 2013.

17. Gray RJ: A class of K-sample tests for comparing the cumulative incidence of a competing risk. Ann Stat 16: 1141-1154, 1988.

18. Fine JP and Gray RJ: A proportional hazards model for the subdistribution of a competing risk. J Am Stat Assoc 94: 496-509, 1999

19. Rosenbaum PR and Rubin DB: Reducing bias in observational studies using subclassification on the propensity score. J Am Stat Assoc 79: 516-524, 1984.

20. Morelli G, Pagni R, Mariani C, et al: Glansectomy with split-thickness skin graft for the treatment of penile carcinoma. Int J Impot Res 21: 311-314, 2009 
21. Feldman AS and McDougal WS: Long-term outcome of excisional organ sparing surgery for carcinoma of the penis. J Urol 186: $1303-1307,2011$

22. O'Kane HF, Pahuja A, Ho KJ, Thwaini A, Nambirajan T and Keane P: Outcome of glansectomy and skin grafting in the management of penile cancer. Adv Urol 2011: 240824, 2011.

23. Li P, Song N, Yin C, et al: Glans-preserving surgery for superficial penile cancer. J Androl 33: 435-440, 2012.

24. Tal R, Jacks LM, Elkin E and Mulhall JP: Penile implant utilization following treatment for prostate cancer: analysis of the SEER-Medicare database. J Sex Med 8: 1797-1804, 2011.

25. Mohs FE, Snow SN, Messing EM and Kuglitsch ME: Microscopically controlled surgery in the treatment of carcinoma of the penis. J Urol 133: 961-966, 1985.

26. Spyropoulos E, Borousas D, Mavrikos S, Dellis A, Bourounis M and Athanasiadis S: Size of external genital organs and somatometric parameters among physically normal men younger than 40 years old. Urol 60: 485-491, 2002.

27. Palminteri E, Berdondini E, Lazzeri M, Mirri F and Barbagli G: Resurfacing and reconstruction of the glans penis. Eur Urol 52: 893-898, 2007.
28. Joura EA: Epidemiology, diagnosis and treatment of vulvar intraepithelial neoplasia. Curr Opin Obstet Gynecol 14: 39-43, 2002.

29. Bezerra AL, Lopes A, Santiago GH, Ribeiro KC, Latorre MR and Villa LL: Human papillomavirus as a prognostic factor in carcinoma of the penis: Analysis of 82 patients treated with amputation and bilateral lymphadenectomy. Cancer 91: 2315-2321, 2001

30. Rubin MA, Kleter B, Zhou M, et al: Detection and typing of human papillomavirus DNA in penile carcinoma: Evidence for multiple independent pathways of penile carcinogenesis. Am J Pathol 159: 1211-1218, 2001.

31. Morris CR, Cohen R, Schlag R and Wright WE: Increasing trends in the use of breast-conserving surgery in California. Am J Public Health 90: 281-284, 2000. 